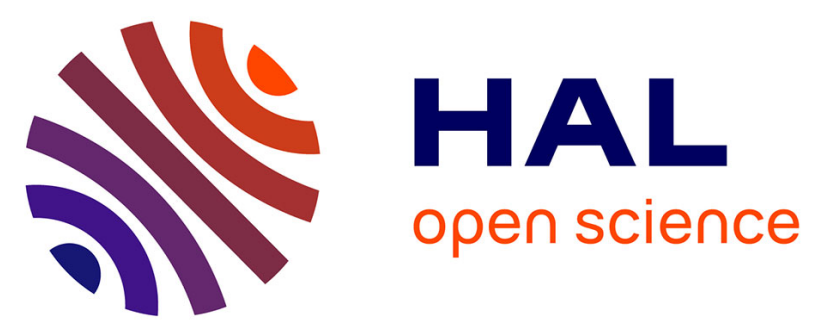

\title{
Croissance estivale en matière sèche de peuplements de fétuque élevée (Festuca arundinacea Schreb.) et de dactyle (Dactylis glomerata L.) dans l'Ouest de la France. I. Etude en conditions de nutrition azotée et d'alimentation hydrique non limitantes
}

\author{
Gilles Lemaire, Alain Denoix
}

\section{To cite this version:}

Gilles Lemaire, Alain Denoix. Croissance estivale en matière sèche de peuplements de fétuque élevée (Festuca arundinacea Schreb.) et de dactyle (Dactylis glomerata L.) dans l'Ouest de la France. I. Etude en conditions de nutrition azotée et d'alimentation hydrique non limitantes. Agronomie, 1987, 7 (6), pp.373-380. hal-00885004

\author{
HAL Id: hal-00885004 \\ https://hal.science/hal-00885004
}

Submitted on 1 Jan 1987

HAL is a multi-disciplinary open access archive for the deposit and dissemination of scientific research documents, whether they are published or not. The documents may come from teaching and research institutions in France or abroad, or from public or private research centers.
L'archive ouverte pluridisciplinaire HAL, est destinée au dépôt et à la diffusion de documents scientifiques de niveau recherche, publiés ou non, émanant des établissements d'enseignement et de recherche français ou étrangers, des laboratoires publics ou privés. 


\title{
Croissance estivale en matière sèche de peuple- ments de fétuque élevée (Festuca arundinacea Schreb.) et de dactyle (Dactylis glomerata L.) dans l'Ouest de la France. I. Etude en conditions de nutrition azotée et d'alimentation hydrique non limitantes
}

\author{
Gilles LEMAIRE \& Alain DENOIX $\left({ }^{*}\right)$ \\ I.N.R.A., Station d'Ecophysiologie des plantes fourragères, Centre de Recherches de Lusignan, \\ F 86600 Lusignan \\ (*) I.N.R.A., Station d'Agronomie, Centre de Recherches d'Angers, F 49000 Angers \\ F.N.I.E., 58, avenue Kléber, F 75784 Paris
}

Pour étudier les effets de la sécheresse sur la croissance estivale de peuplements de graminées, il a en premier lieu été nécessaire de déterminer le « potentiel de croissance » de ces peuplements lorsque la nutrition azotée et l'alimentation hydrique sont non limitantes : des parcelles de fétuque élevée (Festuca arundinacea Schreb.) cv. Clarine et de dactyle (Dactylis glomerata L.) cv. Floreal, convenablement irriguées et recevant de forts apports d'azote (120 kg/ha après la coupe initiale du début de l'été), devaient théoriquement se trouver dans ces conditions.

Néanmoins, la vérification du niveau réel de disponibilité en azote par la méthode des courbes de dilution $\mathrm{N} \%=\alpha(\mathrm{MS})^{-\beta}$ a mis en évidence une insuffisance de nutrition azotée pour 2 années sur les 5 de l'étude. Pour les 3 autres années, on a pu décrire la cinétique de croissance des 2 espèces par un modèle de la forme : $\mathrm{MS}=\mathrm{b}(\mathrm{J}-\mathrm{a})$

où MS représente la matière sèche élaborée au cours de la croissance $(\mathrm{kg} / \mathrm{ha})$ et $\mathrm{J}$ représente le nombre de jours de repousse.

Dans ces conditions, $b$ représente la vitesse de croissance durant la phase linéaire $(\mathrm{kg} / \mathrm{ha} / \mathrm{j})$ et $a$ représente le temps de latence apparent après la coupe en jours.

Des équations semblables ont été obtenues pour les 2 espèces :

MS $=139(\mathrm{~J}-9,2)$ pour la fétuque cv. Clarine

MS $=142(J-10,2)$ pour le dactyle cv. Floreal.

Les faibles différences de conditions climatiques durant les années ayant permis le calage du modèle ne nous permettent pas d'étudier la variabilité de ses coefficients en fonction des paramètres climatiques, température, rayonnement, etc... Néanmoins, il représente une référence stable dans nos conditions et permettra d'apprécier les effets du déficit hydrique et d'un déficit de nutrition azotée sur la croissance estivale. Cet aspect sera abordé dans un second article.

Mots clés additionnels : Courbes de croissance, potentiel de croissance, courbes de dilution de l'azote. limiting nitrogen nutrition and water supply conditions.

To study drought effects on summer regrowth of grass stands, it was first necessary to determine the " growth potential » under non-limiting nitrogen and water conditions. We accordingly studied different plots of tall fescue (Festuca arundinacea Schreb.) cv. Clarine and cocksfoot (Dactylis glomerata L.) cv. Floreal with optimal water supply by irrigation. The plots were uniformly cut, early in summer, and subsequent growth curves were established after an application of $120 \mathrm{~kg} / \mathrm{ha}$ of nitrogen, a level considered as non-limiting. This experiment was conducted over five years. We checked each year if the level of nitrogen nutrition was actually non-limiting, by using the nitrogen dilution model $N \%=\alpha(\mathrm{D} . \mathrm{M})^{-\beta}$.

Despite the high level of nitrogen application, nitrogen shortage appeared to limit summer regrowth for two years out of the five. In the others, it was possible to describe the kinetics of regrowth of both species, in the absence of nitrogen shortage, by a simple model :

$$
\mathrm{D} \cdot \mathrm{M}=b(\mathrm{~J}-a)
$$


were D.M is the dry matter accumulated and $\mathbf{J}$ is the number of days after the cut. Under these conditions, $b$ represents the growth rate during the linear phase $\left(\mathrm{kg} \mathrm{DM} \mathrm{ha}^{-1} \mathrm{day}^{-1}\right)$ and $a$ represents the apparent delay of regrowth after cutting.

Similar equations were obtained for the two cultivars tested : DM $=139(\mathrm{~J}-9.2)$ for tall fescue cv. Clarine ; $\mathrm{DM}=142(\mathrm{~J}-10.2)$ for cocksfoot $\mathrm{cv}$. Floreal. The limited range of climatic variation during the different years did not permit us to study the variability of these coefficients according to temperature, radiation,... This model may be able to provide a stable reference to study the effect of limiting nitrogen and water conditions on summer regrowth. This aspect will be developed in a further paper.

Additional key words : Growth curves, growth potential, nitrogen dilution curves.

\section{INTRODUCTION}

La grande variabilité de la croissance estivale des peuplements prairiaux représente une difficulté essentielle pour l'élaboration des calendriers fourragers prévisionnels. Cette variabilité résulte des conditions de sécheresse estivale qui limitent plus ou moins l'alimentation hydrique des peuplements. A cette cause première peut s'ajouter une variation très importante des niveaux effectifs de nutrition azotée. La prise en compte de l'effet de ces deux facteurs sur la croissance d'une prairie donnée nécessite la détermination préalable de son potentiel de croissance.

Le potentiel de croissance peut être défini comme la croissance d'une prairie, obtenue lorsque tous les facteurs du milieu modifiables par l'agriculteur ont été portés à un niveau non limitant. L'irrigation et la fertilisation permettent de réaliser cette condition pour l'alimentation hydrique, la nutrition azotée et la nutrition minérale $\mathrm{P}$ et $\mathrm{K}$. Cette croissance potentielle peut alors se réaliser et elle est déterminée par les facteurs du milieu non modifiables à l'échelle agricole (température, rayonnement) (MERIAUX \& DELECOLLE, 1980). L'effet d'un déficit d'alimentation hydrique et/ou de nutrition azotée pourra être quantifié par la réduction de la croissance réelle par rapport au témoin représenté par la croissance potentielle. Cette démarche avait déjà été utilisée pour l'étude de la croissance des prairies au printemps (LEMAIRE \& SALETTE, 1981).

La nécessité de se situer à azote non limitant dans une expérimentation au champ nous impose d'avoir une méthode de diagnostic du niveau réel de nutrition azotée du peuplement. Nous utiliserons celle développée dans une étude précédente (SALETTE \& LEMAIRE, 1981) et basée sur l'existence d'une relation d'allométrie entre la dynamique de prélèvement d'azote par les parties aériennes et la dynamique de croissance en matière sèche d'un peuplement de graminées fourragères. Cette relation permet également de rendre compte de la cinétique de diminution des teneurs en azote des parties aériennes au cours de leur croissance, cinétique que nous avions appelée courbe de dilution.

$$
\mathrm{N} \%=\alpha(\mathrm{MS})^{-\beta}
$$

où $\mathrm{N} \%$ représente les teneurs en $\mathrm{N}$ en p. 100 et MS la quantité de matière sèche récoltée en tonne, ha ', $x, \beta$ étant des coefficients.

Cependant, cette relation ayant été établie pour une croissance de printemps, il est nécessaire au préalable d'en vérifier la généralisation pour une croissance estivale afin de pouvoir l'utiliser comme base de diagnos- tic du niveau de nutrition azotée des peuplements étudiés.

Dans ce premier article, nous analyserons la croissance potentielle des 2 espèces. L'effet des déficits d'alimentation hydrique et leur interaction avec la nutrition azotée seront présentés dans un autre article (LEMAIRE \& DENOIX, 1987).

\section{MATÉRIELS ET MÉTHODES}

Pour cette analyse de la croissance estivale, nous disposons de trois séries d'expérimentations sur le site de Lusignan. Le climat de la région est de type océanique avec une sécheresse estivale assez marquée. Le sol est un limon battant superposé à des argiles rouges assez perméables.

Les trois séries d'expérimentations sont décrites dans le tableau 1. Ce sont les mêmes parcelles semées en 1978 qui ont été utilisées pour les expérimentations II et III.

L'analyse de la cinétique de croissance estivale a été entreprise de manière uniforme pour les trois expérimentations, à partir d'une coupe d'homogénéisation à la fin du mois de juin et par des coupes hebdomadaires successives réalisées à la motofaucheuse sur des sousparcelles adjacentes de $6 \mathrm{~m}^{2}$ chacune. A chacune des coupes hebdomadaires, un échantillon d'herbe est prélevé pour la détermination de la teneur en matière sèche et pour l'analyse de la teneur en azote (méthode Kjeldahl), après séchage à $80^{\circ} \mathrm{C}$ et broyage. La cinétique de la repousse est donc exprimée en termes de matière sèche récoltable. Les courbes de croissance en matière sèche et les cinétiques de diminution des teneurs en azote, pour les différents traitements, ont pu être déterminées au cours des coupes successives.

Pour les différentes années, les parcelles étaient homogénéisées à l'automne et au printemps par des coupes et des apports d'azote identiques $(30 \mathrm{~kg}$ par coupe). Sur les traitements irrigués, les apports d'eau étaient réalisés par un dispositif de micro-asperseurs décrit dans une publication précédente (LEMAIRE \& ROBERGE, 1980). Les consommations hydriques de chaque traitement étaient suivies par sonde à neutrons jusqu'à une profondeur de $1,80 \mathrm{~m}$. Les irrigations ont été suffisamment fréquentes pour maintenir la consommation d'eau à un niveau toujours équivalent à l'ETP Penman. Les apports d'azote établis pour la repousse estivale étudiée ont été réalisés immédiatement après la coupe de la fin du printemps. Le tableau 1 résume les différents traitements étudiés. 
TABLEAU 1

Conditions expérimentales et matériel végétal utilisé lors des 3 séries d'expérimentation. Experimental conditions and plant material.

\begin{tabular}{|c|c|c|c|c|c|c|c|}
\hline Série & Année & $\begin{array}{l}\text { Dactyle } \\
\text { cv. }\end{array}$ & $\begin{array}{c}\text { Fétuque } \\
\text { cv. }\end{array}$ & $\begin{array}{c}\text { Régime } \\
\text { hydrique }\end{array}$ & $\begin{array}{l}\text { Date de coupe } \\
\text { fin printemps }\end{array}$ & $\begin{array}{l}\text { Niveaux d'azote } \\
\mathrm{kg} / \mathrm{ha}\end{array}$ & $\begin{array}{l}\text { Nombre de } \\
\text { répétitions }\end{array}$ \\
\hline$I^{*}$ & 1977 & & Ludelle & irrigué & 27 juin & $0-50-100-150$ & 5 \\
\hline \multirow{3}{*}{$\mathrm{II}^{* *}$} & 1979 & Floréal & Clarine & sec/irrigué & 5 juillet & $0-60-120$ & 5 \\
\hline & 1980 & Floréal & Clarine & sec/irrigué & 30 juin & $0-60-120$ & 5 \\
\hline & 1981 & Floréal & Clarine & sec/irrigué & 3 juillet & $0-60-120$ & 5 \\
\hline \multirow{2}{*}{$\mathrm{III}^{* *}$} & 1984 & Floréal & Clarine & sec/irrigué & 25 juin & $60-120$ & 4 \\
\hline & 1985 & Floréal & Clarine & sec/irrigué & 25 juin & $60-120$ & 4 \\
\hline
\end{tabular}

* Semis en avril 1975.

** Semis en avril 1978.

\section{RÉSULTATS ET DISCUSSIONS}

\section{A. Détermination de la référence « azote non limitant»}

L'étude de la réponse de la fétuque élevée à l'azote en 1977 nous permet, étant donné la gamme de niveaux de nutrition azotée testée, de caractériser le niveau non limitant. La figure 1 montre la cinétique de croissance en matière sèche pour les quatre niveaux d'apport d'azote en condition d'alimentation hydrique non limitante. On constate que la croissance en matière sèche du niveau 100 est d'abord identique à celle du niveau 150 , puis très légèrement inférieure dans la deuxième moitié de la période de repousse. L'examen des courbes de dilution (fig. 2) nous confirme qu'à chacun des niveaux d'apport d'azote correspond une courbe déterminée conformément au modèle $\mathrm{N} \%=\alpha(\mathrm{MS})^{-\beta}$.

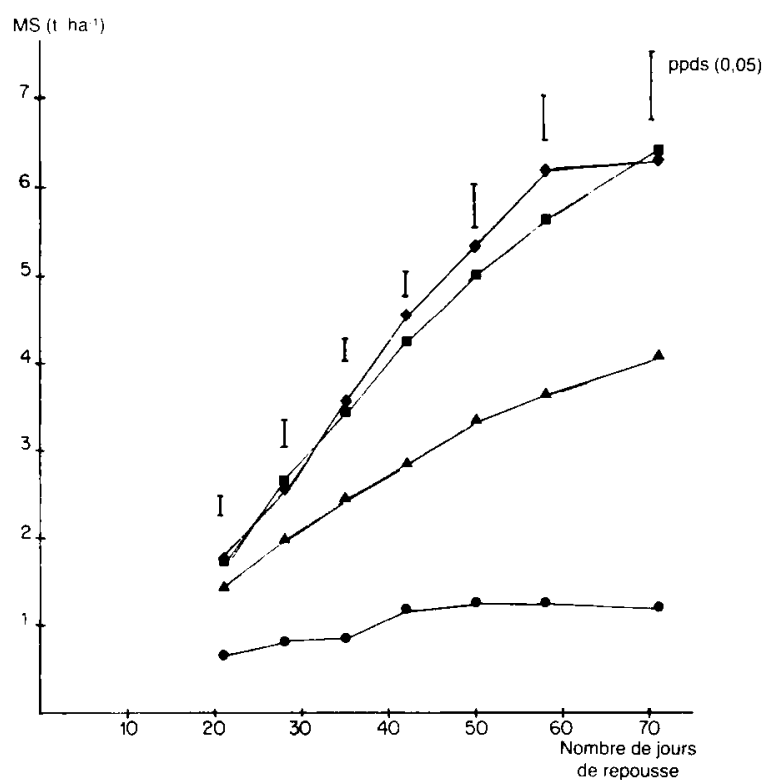

Figure 1

Courbes de croissance estivale en matière sèche (1977) sur la fétuque élevée cv. Ludelle, en fonction de la fertilisation azotée (NO: • N50 : $\boldsymbol{\Delta} ; N 100: \square ; N 150: \bullet)(p p d s:$ plus petite différence significative).

Regrowth curves of tall fescue cv. Ludelle (1977) at different levels of nitrogen application (NO: $\bullet$ N50: $\mathbf{\Delta} ; N 100: \mathbf{E} ; N I 50: \bullet)$ $($ ppds $=L S D=$ Least Significant Difference $)$.

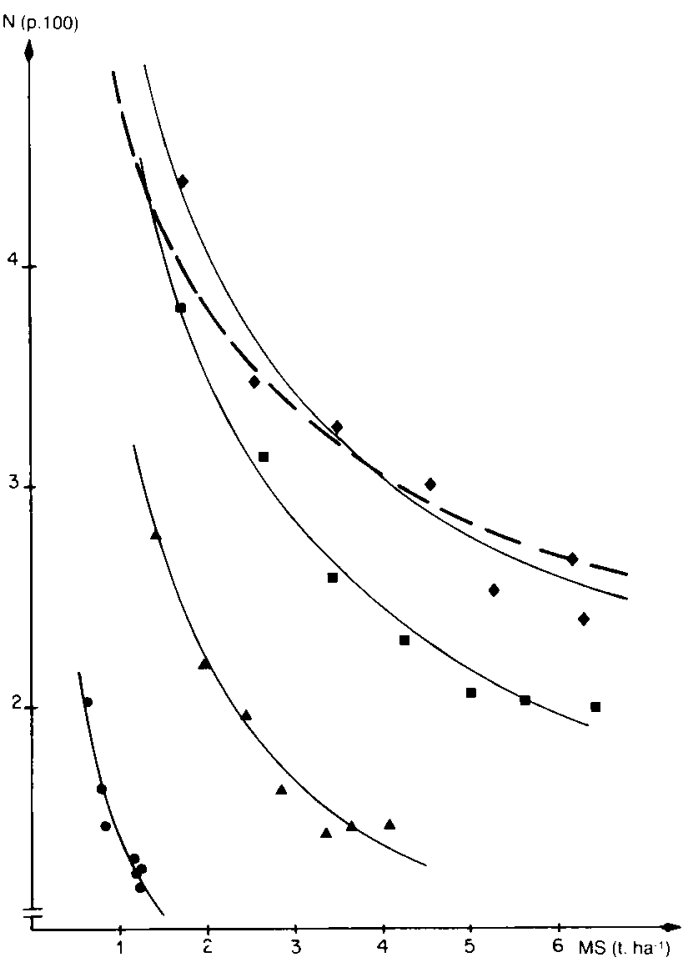

Figure 2

Relations de dilution de l'azote $\left(N \%=\alpha(M S)^{-\beta}\right.$ au cours de la repousse estivale en 1977 sur la fétuque élevée cv. Ludelle, en fonction de la fertilisation azotée

No : $\bullet N \%=1,42(M S)^{-0,72} \quad r=-0,96$

$N 50: \Delta N \%=3,50(M S)^{-0,68} \quad r=-0,98$

N100: $N \%=5,13(M S)^{-0,54} \quad r=-0,97$

N150: $N \%=5,56(M S)^{-0,44} \quad r=-0,98$

- Courbe de référence $N$ non limitant au printemps :

$$
N \%=4,79(M S)^{-0,32} \quad r=-0,98 .
$$

Dilution of nitrogen during summer regrowth $N \%=\alpha(D M)^{-\beta}$ for tall fescue $c v$. Ludelle (1977) at different levels of nitrogen application (NO: $\bullet$; N50: $\mathbf{\Delta} ; N 100: \mathbf{0} ; N 150: \bullet)$.

- - Reference curves for non-limiting nitrogen during spring regrowth.

Nous avons fait figurer la courbe correspondant au niveau de nutrition azotée non limitant pour la croissance de printemps (LEMAIRE \& SALETTE, 1984). Nous constatons que cette courbe se situe à proximité de celle correspondant au niveau 150 ; la courbe correspondant au niveau 100 , proche au départ de la crois- 
sance, diverge par la suite. La courbe de référence de printemps peut donc être utilisée pour décrire le niveau de nutrition azoté non limitant en été : les points $(\mathrm{N} \%, \mathrm{MS})$ situés au-dessus ou à proximité de cette courbe de référence correspondent à des niveaux de nutrition azotée non limitants ou sub-optimum. Par contre, les points qui s'écartent vers des valeurs plus faibles correspondent à des situations où le niveau de nutrition azotée est insuffisant. L'examen simultané des figures 1 et 2 permet d'étalonner la méthode des courbes de dilution comme diagnostic du niveau de nutrition azotée d'un peuplement de graminées : pour une production de matière sèche donnée, la différence entre la teneur en $\mathrm{N}$ mesurée et celle qui correspond à la courbe de dilution de référence ( $\mathrm{N}$ non limitant) peut être considérée comme une indication de l'intensité du déficit de nutrition azotée.

\section{B. Analyse des cinétiques de croissance}

La figure 3 représente les cinétiques de croissance [MS $=f(t)]$ en conditions de forte nutrition azotée par la fétuque élevée (fig. $3 a$ ) et le dactyle (fig. $3 b$ ).

On constate une variabilité entre années pour la production de matière sèche : ainsi, pour la fétuque élevée, après 50 jours de repousse, nous obtenons 4,5 $\mathrm{tha}^{-1}$ de MS en 1979 et $6 \mathrm{tha}^{-1}$ en 1984 ; pour le dactyle, cette variabilité est relativement plus faible : 4,75 t MS en 1979 et 5,7 en 1980.

Pour pouvoir étudier cette variabilité, nous avons utilisé le modèle de croissance linéaire que nous avions précédemment élaboré pour l'étude de la croissance de printemps (LEMAIRE, 1985). En effet, on peut constater, sur les figures 3 ( $a$ et $b$ ), que sur la période de repousse étudiée, la relation entre la MS élaborée et la durée de la repousse est linéaire, si l'on se situe après une certaine période correspondant à la mise en place d'un nouvel appareil photosynthétique. Entre le $15^{\mathrm{e}}$ et le $50^{\mathrm{e}} \mathrm{j}$ de repousse, la croissance peut être représentée par l'équation suivante :

$$
\mathrm{MS}=\mathrm{b}(\mathrm{j}-\mathrm{a})
$$

avec $: j=$ nombre de jours après la coupe

$\mathrm{b}=$ vitesse de croissance en MS $\left(\mathrm{kg} \mathrm{ha}^{-1} \mathrm{j}^{-1}\right)$

$a=$ départ apparent de croissance (nombre de $\mathrm{j}$ après la coupe).

Les ajustements ont été réalisés à partir des points moyens ( 4 ou 5 répétitions selon les années).

Pour chacune des années étudiées et pour chaque espèce, les équations obtenues sont reportées sur le tableau 2. Les vitesses de croissance $b$ varient pour la fétuque élevée de $103 \mathrm{~kg}$ de MS ha-1 $\mathrm{j}^{-1}$ en 1981 à $155 \mathrm{~kg}$ de MS ha ${ }^{-1} \mathrm{j}^{-1}$ en 1984. Pour le dactyle, nous obtenons une variabilité sensiblement plus faible (110 en 1981 à 150 en 1984), mais une bonne concordance avec la fétuque.

Les départs apparents de croissance " a " sont également variables entre années (tabl. 2). Cependant, une part importante de leur variation peut être imputée à la variabilité des vitesses de croissance. En effet, il existe une corrélation positive entre les deux coefficients d'ajustement linéaire $b$ et $a$. La figure 4 permet de mettre en évidence une relation directe entre les valeurs de $a$ et de $b$ pour la majorité des années :

$$
\mathrm{a}=0,169 \mathrm{~b}-14 \quad \mathrm{r}=0,969 \quad(\mathrm{n}=12)
$$
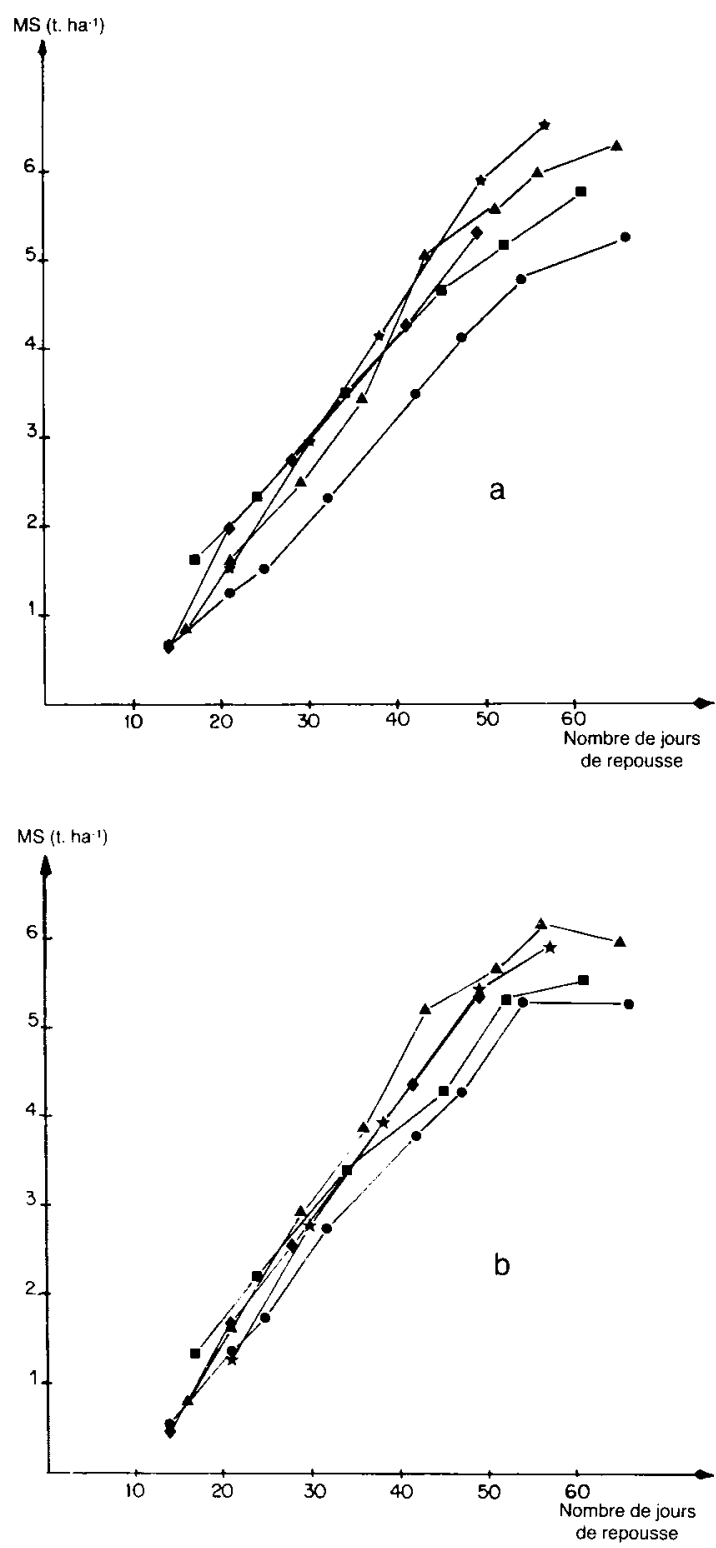

Figure 3

Courbes estivales de croissance en matière sèche de la fétuque élevée $c v$. Clarine (a) et du dactyle cv. Floréal (b) obtenues avec un apport d'azote de $120 \mathrm{~kg} . \mathrm{ha}^{-1}$ après la coupe initiale. (1979: ; 1980 : A ; 1981 : $\mathbf{0} ; 1984: \star ; 1985: \bullet$.)

Regrowth curves of tall fescue cv. Clarine (a) and cocksfoot $\mathrm{cv}$. Floreal (b) with nitrogen application of $120 \mathrm{~kg} . \mathrm{ha}^{-1}$ after an early sum-

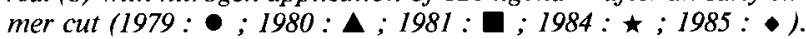

Cette relation permet d'expliquer qu'une croissance rapide pendant la phase linéaire contribue à sousestimer la rapidité de départ en croissance après la coupe et inversement. Ceci provient du fait que la croissance est exponentielle dans les premiers jours de repousse et que nous ne disposons pas de points expérimentaux permettant de caractériser la cinétique de croissance à cette période. En 1979, pour les deux espèces, on constate un retard important dans le départ en croissance non attribuable à une forte vitesse de croissance ultérieure ; au contraire, en 1981, sur la fétuque élevée, on remarque un départ en croissance très rapide. Les causes de ces variations doivent être recherchées dans les conditions de croissance en début de repousse : soit dans des facteurs du milieu, soit dans des facteurs internes au peuplement lui-même (nombre 


\section{TABLEAU 2}

Paramètres de l'équation $M S=b(J-a)$ reliant la matière sèche élaborée (MS) et le nombre de jours de repousse $(J).(r=$ coefficient de corrélation).

Parameters for the relation $M S=b(J-a)$ between accumulated Dry Matter (MS) and number of days of regrowth $(\mathrm{J})$.

\begin{tabular}{|c|c|c|c|}
\hline Année & $\begin{array}{l}\text { Niveaux } \\
\text { d'azote } \\
\mathrm{kg} / \mathrm{ha}\end{array}$ & $\begin{array}{l}\text { Fétuque (1) cv. Ludelle } \\
\text { (2) cv. Clarine }\end{array}$ & Dactyle cv. Floréal \\
\hline \multirow{2}{*}{1977} & 100 & $\begin{aligned} \mathrm{b} & =0,112 \pm 0,009 \\
\mathrm{a} & =4,9 \\
\mathrm{r} & =0,996\end{aligned}$ & \\
\hline & 150 & $\begin{array}{l}\mathrm{b}=0,121 \pm 0,008 \\
\mathrm{a}=6,2 \\
\mathrm{r}=0,998\end{array}$ & \\
\hline 1979 & 120 & $\begin{array}{l}\mathrm{b}=0,107 \pm 0,006 \\
\mathrm{a}=9,1 \\
\mathrm{r}=0,998\end{array}$ & $\begin{array}{l}b=0,118 \pm 0,005 \\
a=9,7 \\
r=0,999\end{array}$ \\
\hline 1980 & 120 & $\begin{array}{l}\mathrm{b}=0,134 \pm 0,015 \\
\mathrm{a}=9,3 \\
\mathrm{r}=0,992\end{array}$ & $\begin{array}{l}\mathrm{b}=0,138 \pm 0,017 \\
\mathrm{a}=8,6 \\
\mathrm{r}=0,991\end{array}$ \\
\hline 1981 & 120 & $\begin{array}{l}\mathrm{b}=0,103 \pm 0,007 \\
\mathrm{a}=0,7 \\
\mathrm{r}=0,998\end{array}$ & $\begin{array}{l}b=0,110 \pm 0,009 \\
a=4,2 \\
r=0,997\end{array}$ \\
\hline 1984 & 120 & $\begin{array}{l}\mathrm{b}=0,155 \pm 0,006 \\
\mathrm{a}=11 \\
\mathbf{r}=0,999\end{array}$ & $\begin{array}{l}\mathrm{b}=0,150 \pm 0,023 \\
\mathrm{a}=12,1 \\
\mathrm{r}=0,998\end{array}$ \\
\hline 1985 & 120 & $\begin{array}{l}\mathbf{b}=0,127 \pm 0,023 \\
\mathrm{a}=7,2 \\
\mathrm{r}=0,995\end{array}$ & $\begin{array}{l}\mathrm{b}=0,137 \pm 0,013 \\
\mathrm{a}=9,8 \\
\mathrm{r}=0,999\end{array}$ \\
\hline
\end{tabular}

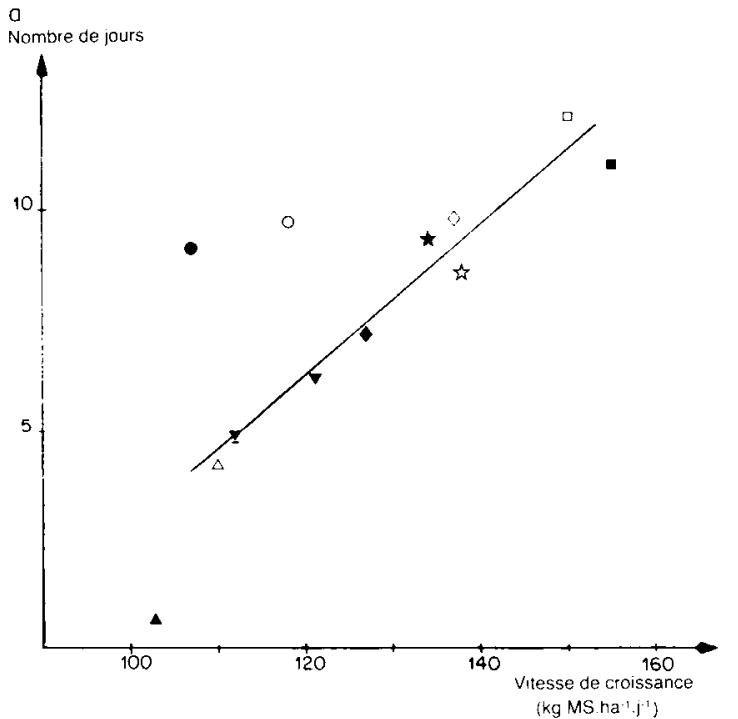

Figure 4

Relation entre les 2 paramètres a et $b$ du modèle : $M S=b(J-a)$, avec des niveaux d'apport d'azote élevés, pour la fétuque (1977:

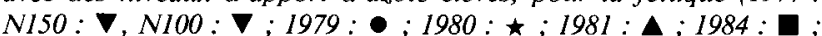
$1985: \diamond)$ et le dactyle $(1979: 0 ; 1980:$ 故 $1981: \triangle ; 1984: \square$; $1985: \diamond)$.

Relationship between parameters $a$ and $b$ of equation $D M=b$ $(J-a)$ representing dry matter accumulation (DM) after cutting. $b=$ growth rate $\left(\mathrm{kg} \cdot \mathrm{ha}^{-1} \cdot \mathrm{j}^{-1}\right)$,

$J=$ number of days after cutting,

$a=$ apparent delay of regrowth.

Parameters obtained for the different years $(1977: \nabla ; 1979$ : $\bullet \bigcirc ; 1980: \star$ is ; 1981: $\Delta \triangle ; 1984: \mathbf{\square} \square ; 1985: \diamond \diamond)$ for tall fescue (black symbols) and cocksfoot (white symbols). de talles, niveau des réserves glucidiques, etc...), qui accélèrent ou retardent la mise en place des premières feuilles. L'absence de mesures précises de l'évolution de ces paramètres dans les premiers jours de la repousse ne nous permet pas d'aller plus loin dans cette analyse.

Cependant, avant de vouloir interpréter l'effet du climat sur la variabilité constatée sur les vitesses de croissance et les départs apparents, il est indispensable de vérifier que le niveau réel de nutrition azotée était bien non limitant pour chacune des années étudiées.

\section{Analyse du niveau de nutrition azotée}

La figure 5 représente les courbes de dilution de l'azote dans la matière sèche obtenues sur la fétuque (fig. $5 a$ ) et sur le dactyle (fig. $5 b$ ) pour les différentes années, après un apport de $120 \mathrm{~kg} \mathrm{~N} \mathrm{ha}^{-1}$. Nous avons également fait figurer la courbe de référence $\mathrm{N}$ non limitant. Nous constatons qu'en 1979 et 1981 le niveau de nutrition azotée a été limitant pour la croissance en matière sèche. Ceci peut être mis en relation avec les faibles vitesses de croissance obtenues ces années-là (107 et $103 \mathrm{~kg} \mathrm{ha}^{-1} \mathrm{j}^{-1}$ de MS respectivement pour la fétuque) comparativement aux trois autres années. En 1981, le niveau de nutrition azotée initial était pourtant satisfaisant, mais il s'est rapidement dégradé au cours de la croissance. On peut d'ailleurs constater en se reportant à la figure 3 que cette baisse du niveau de nutrition azotée s'est manifestée par un ralentissement de la croissance en fin de repousse. En 1979, au contraire, la nutrition azotée a été très limitante dès le début de la repousse, ce qui peut expliquer le retard important du départ apparent de la croissance, observé cette année-là (fig. 3). En 1980, le niveau de nutrition azotée reste optimum pour la fétuque pendant la plus grande partie de la repousse, et ne diminue que lorsque une production de $4 \mathrm{tha}^{-1}$ a été obtenue. Cette restriction de l'offre en azote à ce stade de repousse n'a eu que peu d'effet sur les courbes de croissance. Néanmoins, nous nous sommes limités aux 50 premiers jours de repousse pour le calcul du potentiel de croissance.

Les courbes obtenues sur le dactyle confirment les observations faites sur la fétuque. Nous n'observons cependant pas de perturbation de la nutrition azotée en fin de croissance en 1980 : ceci est probablement dû au fait que le dactyle a une plus grande capacité que la fétuque à prélever l'azote du sol en situation de plus faible disponibilité (LEMAIRE \& DENOIX, 1987).

Les restrictions de disponibilité en azote observées en 79 et 81 sur les 2 espèces doivent donc provenir d'un phénomène lié à l'année pour lequel nous formulons l'hypothèse d'une interaction avec la dynamique de l'azote dans le sol : la conduite de l'irrigation telle qu'elle a été réalisée (contrôles de l'humidité du sol avec la sonde à neutrons) ne permet pas de penser qu'il $y$ ait eu entraînement de l'eau et donc de $\mathrm{NO}_{3}^{-}$au-delà de la profondeur exploitée par les racines. On peut alors émettre l'hypothèse d'une immobilisation de l'azote minéral apporté, par la biomasse microbienne. Il est intéressant de noter qu'en 1979, avec un apport de $120 \mathrm{~kg} \mathrm{~N}$, le niveau de nutrition azotée était voisin de celui obtenu avec un apport de $60 \mathrm{~kg}$ seulement en 1980 (LEMAIRE \& DENOIX, 1987). Cet exemple illustre 


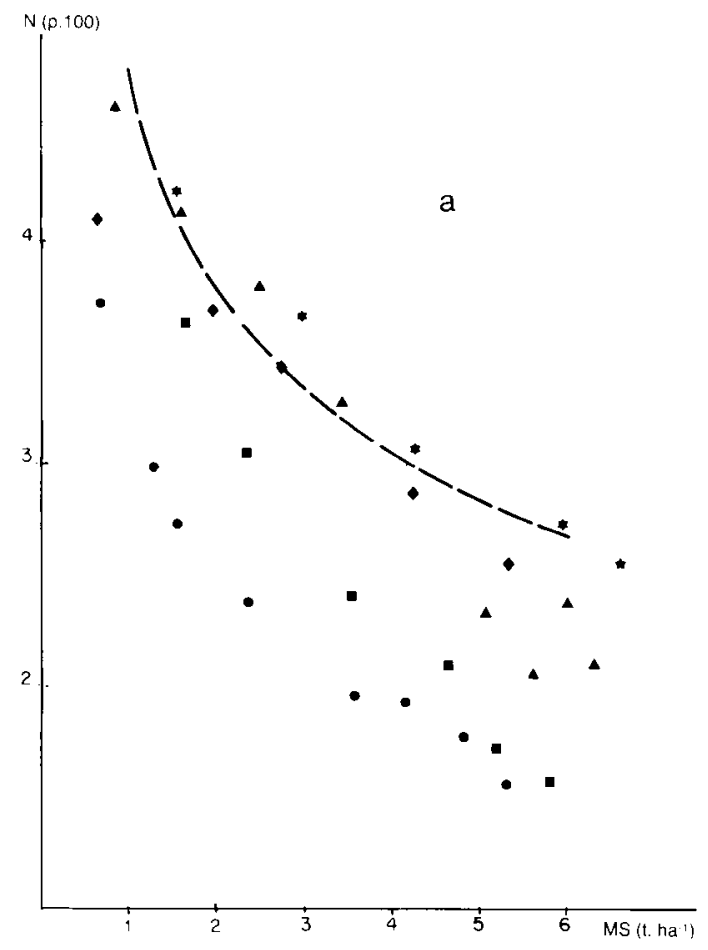

Figure 5

Relations de dilution de l'azote $N \%=\alpha(M S)^{-\beta}$ au cours de la repousse estivale pour la fétuque élevée cv. Clarine (a) et de dactyle cv. Floreal (b) après application de $120 \mathrm{~kg} \mathrm{~N} . \mathrm{ha}^{-1}$ ét pour les différentes années $(1979: \bullet ; 1980: \boldsymbol{\Delta} ; 1981: \mathbf{\square} ; 1984: \star ; 1985:$ $\bullet)$.

- Courbe de référence $N$ non limitant.

parfaitement la nécessité d'un tel diagnostic du niveau réel de nutrition azotée pour l'interprétation correcte des résultats expérimentaux.

\section{Détermination du potentiel estival de croissance en matière sèche}

A partir des 5 années de résultats sur la fétuque élevée $\mathrm{cv}$. Clarine et le dactyle cv. Floréal dont nous disposions initialement pour étudier le potentiel de croissance estivale, il ne reste plus que les 3 années 1980, 84 et 85 pour lesquelles nous pouvons être certains d'avoir été à la fois en conditions hydriques et de nutrition azotée non limitantes.

Pour la fétuque élevée cv. Clarine, nous obtenons sur ces 3 années un potentiel de croissance moyen qui peut être exprimé par l'équation suivante :

$$
\text { MS }=0,139(\mathrm{~J}-9,2)
$$

soit une vitesse de croissance de $139 \mathrm{~kg} \mathrm{MS} \mathrm{ha}^{-1} \mathrm{j}^{-1}$ représentant une production de $2,94 \mathrm{t} \mathrm{MS} \mathrm{ha}^{-1}$ en $30 \mathrm{j}$ ou de 5,7 en $50 \mathrm{j}$ de repousse.

Pour le dactyle cv. Floréal, le potentiel moyen est de :

$$
\text { MS }=0,142(\mathrm{~J}-10,2)
$$

soit une vitesse de croissance de $142 \mathrm{~kg} \mathrm{MS} \mathrm{ha}^{-1} \mathrm{j}^{-1}$ représentant une production de $2,8 \mathrm{t} \mathrm{MS} \mathrm{ha}^{-1}$ en $30 \mathrm{j}$ ou de $5,7 \mathrm{t}$ en $50 \mathrm{j}$ de repousse.

Nous constatons qu'il n'y a aucune différence significative de potentialité de croissance entre ces 2 culti-

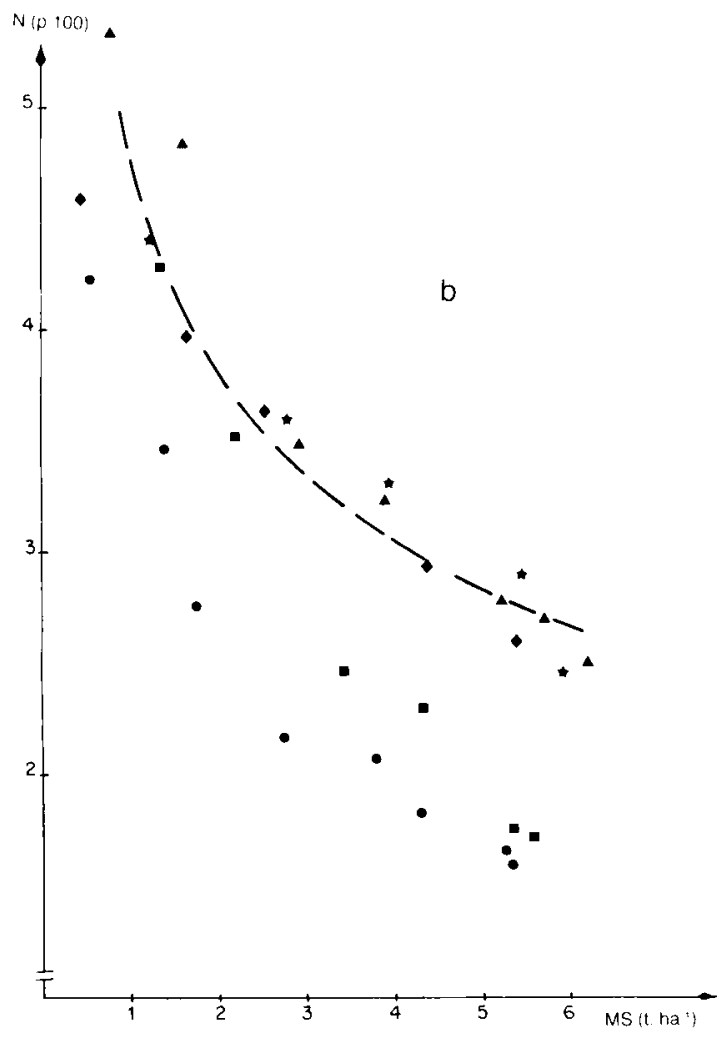

Dilution of nitrogen during summer regrowth $N \%=\alpha(D M)^{-\beta}$ for tall fescue $c v$. Clarine (a) and cocksfoot $c v$. Floreal (b) for the different years with an application of $120 \mathrm{~kg} . \mathrm{ha}^{-1}$ of nitrogen $(1979$ : $\bullet ; 1980: \mathbf{\Delta} ; 1981: \mathbf{\square} ; 1984: \star ; 1985: \bullet)$.

- - Reference curve for non-limiting nitrogen nutrition.

vars. On ne peut toutefois en tirer aucune conclusion quant aux potentialités respectives des 2 espèces dans leur ensemble.

La comparaison entre les 3 années 1980,84 et 85 peut être effectuée à partir des régressions communes aux 2 espèces. Nous obtenons :

$\begin{array}{lllll}1980 & \text { MS }=0,136 \pm 0,012 & (\mathrm{~J}-9,0) & \mathrm{r}=0,990 & \mathrm{n}=14 \\ 1984 & \text { MS }=0,152 \pm 0,015 & (\mathrm{~J} \pm 11,5) & \mathrm{r}=0,995 & \mathrm{n}=8 \\ 1985 & \text { MS }=0,132 \pm 0,010 & (\mathrm{~J} \pm 8,6) & \mathrm{r}=0,996 & \mathrm{n}=10\end{array}$

La comparaison des pentes 2 à 2 entre les années par un test de Student nous permet de mettre en évidence une différence de vitesse de croissance entre 1984 et 1985 ( $\mathrm{t}$ obs. $=2,63 ; \mathrm{t} 0,05=2,15) ;$ par contre 1980 ne se différencie pas significativement de 1984 ( $\mathrm{t}$ obs. $=1,54 ; \mathrm{t} 0,05=2,10)$.

Cette vitesse de croissance plus forte en 1984 n'a pu être reliée à des conditions climatiques particulières : le tableau 3 montre que l'année 1980 se caractérise par des températures plus fraîches en juillet, mais aucune différence notable n'apparaît entre les années 1984 et 85.

A partir de cette étude, on peut donc estimer que les équations (1) et (2) représentent effectivement le potentiel de croissance de la fétuque élevée cv. Clarine et du dactyle cv. Floréal, dans les conditions climatiques précisées. On ne peut, à partir de ces résultats, extrapoler à d'autres conditions plus variables. Cependant, ces potentiels peuvent être comparés à ceux qui ont été déterminés par différents auteurs. Ainsi, pour la même variété de dactyle cv. Floréal, MERIAUX $(1965,1977)$ 
TABLEAU 3

Comparaison des conditions climatiques des années 1980, 84 et 85 . Climatic conditions for years 1980, 84 and 85 .

\begin{tabular}{|c|c|c|c|c|c|c|c|c|c|c|}
\hline & & \multicolumn{3}{|c|}{1980} & \multicolumn{3}{|c|}{1984} & \multicolumn{3}{|c|}{1985} \\
\hline & & $\mathrm{T}$ moy & $\mathbf{P}$ & ETP & $\mathrm{T}$ moy & $\mathrm{P}$ & ETP & $\mathrm{T}$ moy & $\mathrm{P}$ & ETP \\
\hline \multirow{3}{*}{$\begin{array}{l}\mathrm{J} \\
\mathrm{U} \\
\mathrm{I} \\
\mathrm{L} \\
\mathrm{L} \\
\mathrm{E} \\
\mathrm{T}\end{array}$} & $0-10$ & 15,1 & 15,6 & 31,6 & 19,4 & 0,0 & 52,3 & 20,1 & 12,2 & 50,1 \\
\hline & $10-20$ & 15,5 & 41,8 & 30,3 & 18,5 & 3,1 & 44,9 & 19,6 & 4,2 & 47,0 \\
\hline & $20-31$ & 19,1 & 7,7 & 44,8 & 20,9 & 43,5 & 49,7 & 19,4 & 30,0 & 44,4 \\
\hline \multirow{2}{*}{$\begin{array}{l}\mathrm{A} \\
\mathrm{O} \\
\mathrm{U} \\
\mathrm{T}\end{array}$} & $0-10$ & 20,1 & 2,4 & 38,7 & 17,3 & 14,3 & 30,8 & 16,8 & 8,4 & 33,7 \\
\hline & $10-20$ & 18,5 & 5,9 & 30,3 & 19,1 & 0,2 & 38,4 & 17,9 & 4,6 & 31,9 \\
\hline TOTAL & & & 73,4 & 175,7 & & 61,1 & 216,1 & & 59,4 & 207,1 \\
\hline
\end{tabular}

$\mathrm{T}$ moy : température moyenne, en ${ }^{\circ} \mathrm{C}$; mean temperature, in ${ }^{\circ} \mathrm{C}$.

$P \quad$ : pluie, en $\mathrm{mm}$; rainfall, in $\mathrm{mm}$.

ETP : évapotranspiration potentielle, en $\mathrm{mm}$; potential evapotranspiration, in $\mathrm{mm}$.

signale une production maximum de $4,4 \mathrm{tha}^{-1}$ de MS pour une repousse de 40 jours. L'estimation de cette production à partir de l'équation (2) nous donne un résultat tout à fait comparable de $4,2 \mathrm{t}$. Sur la fétuque élevée, MARTY et al. (1977) signalent des vitesses de croissance très inférieures à celles que nous avons obtenues : 70 à $80 \mathrm{~kg} \mathrm{MS} \mathrm{ha}^{-1} \mathrm{j}^{-1}$. Cependant, il n'est pas absolument certain que ces auteurs se soient placés dans des conditions de nutrition azotée non limitante. En outre, ils ont mis en évidence des effets négatifs des fortes températures et des fortes valeurs de l'ETP sur les capacités de croissance estivale. MERIAUX (1980) et MERIAUX \& DELECOLLE (1980) signalent également ce phénomène sur la fétuque élevée $\mathrm{cv}$. Manade pour laquelle une réduction de la croissance était observée lorsque les températures maximales dépassaient $26^{\circ} \mathrm{C}$ et que l'humidité de l'air descendait en dessous de 40 p. 100 durant la journée. Dans les conditions de Lusignan, ces circonstances climatiques sont cependant peu fréquentes.

Une expression plus générale du potentiel de croissance estivale des différents génotypes de graminées fourragères pourrait être obtenue en utilisant les relations entre la matière sèche accumulée dans les parties aériennes et la somme du rayonnement intercepté par le couvert végétal (GOSSE et al., 1986). Pour les graminées fourragères, cette relation n'a été établie que pour des repousses de printemps en période reproductrice (LEMAIRE et al., 1984). Une telle analyse, nécessitant la détermination de la cinétique d'évolution de l'indice foliaire, n'a pu être entreprise et fera l'objet d'une étude ultérieure.

\section{CONCLUSIONS}

Cette étude a permis de montrer qu'il était possible de caractériser le potentiel estival de croissance d'une graminée fourragère par un modèle linéaire reliant directement l'accumulation de matière sèche dans les parties aériennes au nombre de jours de repousse. Apparemment, dans les conditions climatiques où nous avons travaillé, cette relation semble suffisamment stable pour servir de modèle de référence. On peut penser que les paramètres de l'équation pourraient être assez sensiblement modifiés dans des situations climatiques caractérisées par des températures maximales et des ETP journalières plus élevées.

La démarche entreprise a cependant fait ressortir un point méthodologique très important. En effet, la caractérisation du potentiel de croissance nécessite la certitude du maintien du peuplement végétal dans des conditions non limitantes de nutrition azotée. Nous avons pu observer que, malgré des apports d'azote importants en début de repousse, cette condition n'a pas toujours été assurée pour chaque année, tout au long de la repousse. Lorsque les conditions optimales sont réalisées, la dynamique de croissance obtenue peut constituer une référence permettant de quantifier les effets dépressifs d'une diminution à la fois de la disponibilité en azote et de l'alimentation hydrique. C'est cette deuxième analyse qui est présentée dans un autre article. 


\section{RÉFÉRENCES BIBLIOGRAPHIQUES}

Gosse G., Varlet-Grancher C., Bonhomme R., Chartier M., Altirand J. M., Lemaire G., 1986. Production maximale de matière sèche et rayonnement solaire intercepté par un couvert végétal. Agronomie, 6 (1), 47-56.

Lemaire G., 1985. Cinétique de croissance d'un peuplement de fétuque élevée (Festuca arundinacea Schreb.) pendant l'hiver et le printemps. Effets des facteurs climatiques. Thèse de doctorat d'état, Univ. Caen, $96 \mathrm{p}$.

Lemaire G., Denoix A., 1987. Croissance estivale en matière sèche de peuplements de fétuque élevée et de dactyle dans l'ouest de la France. II. Interaction entre les niveaux d'alimentation hydrique et de nutrition azotée. Agronomie, 7 (6), 381-389.

Lemaire G., Roberge G., 1980. Mise au point d'un dispositif expérimental permettant de suivre la consommation hydrique d'une prairie au cours de sa croissance. Ann. agron., 31 (4), 455-471.

Lemaire G., Salette J., 1981. Analyse de l'influence de la température sur la croissance de printemps de graminées fourragères. $C$. $R$. hebd. Séances Acad. Sci., 292, 843-846.

Lemaire G., Salette J., 1984. Relation entre dynamique de croissance et dynamique de prélèvement d'azote pour un peuplement de graminées fourragères. I. Etude de l'effet du milieu. Agronomie, 4 (5), 423440 .
Lemaire G., Gosse G., Chartier M., 1984. Modelling or the dry matter regrowth of a grass stand in spring. Interaction between climatic factors and nitrogen nutrition. Proc. 10th General Meeting or Eur. Grassland Fed. As Norway. Ed. The Norvegian State Agric. Res. Sta., 515-519.

Marty J. R., Bosc N., Hilaire A., 1977. Production comparée de quelques espèces fourragères en condition irriguée ou non. Efficience et valorisation de l'eau. Coûts de production. Fourrages, 70, 91-120.

Meriaux S., 1965. Réactions du dactyle aux facteurs climatiques en fonction du déficit hydrique et de la nutrition azotée. Fourrages, 21, 33-43.

Meriaux S., 1977. Principes de conduite de l'irrigation des plantes fourragères. Fourrages, 70, 43-61.

Meriaux S., 1980. Effects of climate on the yield response of two fescue species to water and nitrogen application. Irrig. Sci., 1, 233-239.

Meriaux S., Delecolle R., 1980. Hiérarchie entre les facteurs physiques du milieu intervenant sur le taux de conversion de l'énergie solaire par quelques graminées fourragères. C. R. Acad. Agric. Fr., 66 (18), 1559-1575.

Salette J., Lemaire G., 1981. Sur la variation de la teneur en azote de graminees fourrageres pendant leur croissance : formulation d'une loi de dilution. C. R. hebd. Séances Acad. Sci., 292, 985-878. 